\title{
Simplicity, Language-Dependency and the Best System Account of Laws*
}

\author{
Billy WHEELER
}

Received: 29/05/2015

Final Version: 13/10/2015

BIBLID 0495-4548(2016)31:2p.189-206

DOI: $10.1387 /$ theoria. 14558

ABSTRACT: It is often said that the best system account of laws (BSA) needs supplementing with a theory of perfectly natural properties. The 'strength' and 'simplicity' of a system is language-relative and without a fixed vocabulary it is impossible to compare rival systems. Recently a number of philosophers have attempted to reformulate the BSA in an effort to avoid commitment to natural properties. I assess these proposals and argue that they are problematic as they stand. Nonetheless, I agree with their aim, and show that if simplicity is interpreted as 'compression', algorithmic information theory provides a framework for system comparison without the need for natural properties.

Keywords: laws of nature; best system account; natural properties; algorithmic information theory; invariance theorem.

RESUMEN: A menudo se dice que la explicación de las leyes del mejor sistema (BSA) requiere ser completada con una teoría de las propiedades perfectamente naturales. La 'fuerza' y la 'simplicidad' de un sistema son relativas a un lenguaje y sin un vocabulario fijo es imposible comparar sistemas rivales. Recientemente, varios filósofos han intentado reformular la BSA en un esfuerzo por evitar el compromiso con las propiedades naturales. Aquí valoro estas propuestas y argumento que son problemáticas en su forma actual. Sin embargo, comparto su objetivo y muestro que si la simplicidad es interpretada como 'compresión', la teoría algorítmica de la información proporciona un marco para la comparación sin necesidad de apelar a propiedades naturales.

Palabras clave: Leyes de la naturaleza, explicación del mejor sistema, propiedades naturales, teoría algorítmica de la información, teorema de invariancia.

\section{Introduction}

The best system account (BSA) is one of the most widely adopted theories of laws. It has been appealing for philosophers with broadly empiricist or Humean sympathies as it analyses the concept of a law of nature without needing to appeal to necessary connections, powers, essences-or any other irreducibly modal concept. The BSA defines the laws as the axioms (and theorems) in a deductive system of all the actual and future empirical truths in our world. As David Lewis $(1973,1983,1986,1994)$, one of the BSA's main defenders noted, deductive systems can be more or less simple and more or less informative: it is reasonable to assume that we value these in equal measure, so that the ideal or 'best system' is

* Part of the research for this paper was funded by the UK Arts and Humanities Research Council (133769). I am indebted to Tim Lewens, Alex Broadbent, Mark Sprevak, Helen Beebee and Anna Alexandrova for discussion and advice on earlier drafts of this text. I am also grateful to two anonymous referees. 
the one which best balances as much of each as possible. One of the BSA's selling points is that it seems to offer a more-or-less objective account of lawhood. Although we have not yet discovered all the empirical truths for our world, we can imagine an ideal systematization of them, and identify the laws of nature as the axioms in that system.

Despite this apparent benefit, it was soon realized that the simplicity measure on systems is language-dependent so that one cannot say that one system is simpler than a rival without first agreeing on the predicate terms the system should be couched it (Lewis 1983; Armstrong, 1983). But who is to decide?

Lewis (1983) solved this problem by making a distinction between the kinds of properties that exist. Not all properties are equal, some-the natural properties-form a privileged set. According to Lewis natural properties provide a simple solution to the language-dependency of simplicity: place as a restriction on all candidate systems that they are formulated only in predicates that refer to perfectly natural properties.

In the time since Lewis offered this solution there has been a growing dissatisfaction among contemporary Humeans. After all, although not strictly modal in character, natural properties seem mysterious and sit uncomfortably with the traditional Humean standpoint of eschewing unobservable metaphysical primitives. To this end a number of reformulations of the BSA have recently been proposed that claim to solve the language-dependency problem whilst simultaneously avoiding the need to postulate natural properties.

This paper will discuss in detail one class of natural-property-free solutions that we might call 'adaptivist'. I take Barry Loewer's (2007) 'package deal account' and Jonathan Cohen and Craig Callender's (2009) 'better best system account' as prime examples of this approach, although others could have been given as well. ${ }^{1}$ The core idea behind adaptivist versions of the BSA is that lawhood is language-relative; however, science will eventually converge upon the 'right language' under internal pressure on theory design from pragmatic and explanatory considerations. It is assumed that these standards on a good theory are more-or-less universal permitting system comparison regardless of the language used.

My criticism of current adaptivist approaches is that they fail to do two things: (i) identify clearly which pragmatic and explanatory considerations are important to science, and (ii) demonstrate how the sharing of these standards can be used to decide among empirically equivalent theories composed of alternative languages.

I agree that adaptivism is the right response to the language-dependency problem and so to overcome the concerns raised in section 3 will propose one theoretical virtue that can fulfill these two aims. I shall argue that one dimension to 'simplicity' that scientists clearly care about is that of compression: good theories compress their empirical data sets. In section 4 I give evidence for thinking why compressibility is an important factor in theory design and evaluation and in section 5 give an account of how it could be used to decide among competing theories.

First I will start by looking in more detail at how the language-dependency problem gets generated and the reasons why some Humeans have become dissatisfied with Lewis' original solution.

1 Similar approaches have also been taken by John Halpin (2003), Brian Taylor (1993), John Roberts (1998) and Markus Schrenk (2008). However, see Eddon and Meacham (2015) for a more finegrained classification of solutions. 


\section{The Language-Dependency Problem}

The realization that the simplicity of a system is dependent on the terms used to construct it was highlighted early in its current form (Lewis 1983; Armstrong 1983). Lewis doesn't say much about what the simplicity or strength of a system consists in. One intuitive interpretation (that Lewis tacitly appeals to on a number of occasions) is to equate the simplicity of a system with the length and number of the axioms, and the strength of a system, with the number of possible worlds it excludes. Defined like this, it is easy to appreciate why the same system could be made simpler just by a change of language. Lewis gives a very simple argument to demonstrate this point:

In fact, the content of any system whatever may be formulated very simply indeed. Given system $S$, let $\mathrm{F}$ be a predicate that applies to all and only things at the world where $S$ holds. Take $F$ as primitive, and axiomatise $S$ (or an equivalent thereof) by the single axiom $(\mathrm{x})(\mathrm{Fx})$. If utter simplicity is so easily attained, the ideal theory may as well be as strong as possible. Simplicity and strength needn't be traded off. Then the ideal theory will include (its simple axiom will strictly imply) all truths, and a fortiori all regularities. Then, after all, every regularity will be a law. That must be wrong. $(1983,367)$

Since a 'best system' can be so easily had it runs the risking of conflating the law/accident distinction therefore making the BSA useless as a theory of laws.

One might want to argue that no serious scientist would consider $(\mathrm{x})(\mathrm{Fx})$ a genuine law, because although it may be simple, it isn't very strong. The original system $S$ tells us more about our world than ' $\mathrm{F}$ ' and so gives us 'more information'. The problem with this type of response, as Armstrong has noted $(1983,68)$, is that what counts as a strong system also depends on how one 'carves nature'. On Lewis' formal definition, $(\mathrm{x})(\mathrm{Fx})$ is as strong as it can be, since it picks out the actual world and only the actual world. However, if by 'strength' we instead mean the sorts of physical systems scientists are actually interested in - such as atoms, quarks, strings and their properties - then we are already assuming a metaphysical partitioning of the world, one which the advocate of ' $F$ ' could simply deny as irrelevant.

Lewis' own solution is to place a restriction on the language for candidate systems and his preferred way of doing this is to posit the existence of perfectly natural properties:

The remedy, of course, is not to tolerate such a perverse choice of primitive vocabulary. We should ask how candidate systems compare in simplicity when each is formulated in the simplest eligible way; or, if we count different formulations as different systems, we should dismiss the ineligible ones from candidacy. An appropriate standard of eligibility is not far to seek: let the primitive vocabulary that appears in the axioms refer only to perfectly natural properties. $(1983,216)$

Lewis is a nominalist concerning the nature of properties. More specifically, he is a classnominalist equating properties with sets of actual and possible individuals $(1983,1986)$. This view stands in opposition to universals and tropes, which unlike properties on Lewis' view, provide the ground for their own existence. Another difference between class-nominalism and universal or trope theories is their size. Whereas there is only a finite number of universals and tropes, class-nominalism implies an infinite number of properties-one for every grouping imaginable. 
The abundance of properties implied by class-nominalism makes the sharing of properties unsuitable for use in metaphysical analysis (trivially all individuals both share and lack an infinite number of properties). Because of this, Lewis justifies the addition of a sparse/ abundant distinction to his view on largely pragmatic grounds: he says natural properties can 'earn their living' by doing 'much needed work'. In particular they can provide a systematic and unified treatment of topics as diverse as duplication; supervenience; divergent worlds; a minimal form of materialism; laws; causation; counterfactuals and reference, adding 'perhaps this list could be extended' (1983, 188-189).

With concerns to the current problem, the appeal is of immediate benefit since it simply rules out the system $(\mathrm{x})(\mathrm{Fx})$ on the grounds that $\mathrm{F}$ does not refer to a natural property.

Despite the ease with which natural properties could solve the problem of languagedependency, many contemporary Humeans remain unsatisfied. Brain Taylor complains 'I confess to finding the joints utterly mysterious, the manner of the carving entirely arcane. Systematic theorists do better, I suggest, to eschew such esoteria' (1993, 88). Barry Loewer is equally scathing: 'Lewis' metaphysical notion of natural property is not needed by the BSA and, in fact, undermines what seems to me the most attractive feature of the BSA' $(2007,316)$.

Why are contemporary Humeans so unhappy with Lewis' appeal to natural properties? On the one hand the distinction Lewis makes seems sensible. We all mostly agree that we should describe the world in terms of 'green' and 'blue' and not Goodman's 'grue' or 'bleen'. Surely the best explanation for this is that 'blue' and 'green' refer to genuine distinctions in kinds whereas 'grue' and 'bleen' do not. One worry concerns what it is that grounds this distinction in the world. We have seen that Lewis' class-nominalism alone cannot accommodate such a distinction. Lewis remains non-committed to what makes a property 'natural', i.e. whether it is taken as an irreducible aspect of classes or is supplied by universals or tropes $(1983,194)$. What does seem necessary for Lewis is that it is taken as a metaphysical primitive.

The main reason why some are unhappy with Lewis' appeal here is not down to metaphysical reasons but mostly epistemological ones. Those who follow Hume in thinking observation should guide one's metaphysics question whether we could ever be in a position to know whether a given property $\mathrm{F}$ is natural or not. Natural properties seem to fail what John Earman (1984) has called 'the empiricist loyalty test'. This can be illustrated with Armstrong's non-Humean theory of laws. According to Armstrong (1983), 'Fs are Gs' is a law of nature when there exists a second-order relation of necessitation between two firstorder universals: represented as $\mathrm{N}(\mathrm{F}, \mathrm{G})$. Earman complains that a world in which $\mathrm{N}(\mathrm{F}, \mathrm{G})$ obtains would be no different observationally than a world in which only $(\mathrm{x})(\mathrm{Fx} \supset \mathrm{Gx}) \mathrm{ob}$ tains. Therefore, there are no good epistemological reasons for postulating the existence of . $^{2}$

Bas van Fraassen (1989) gives a convincing story for thinking that the empiricist loyalty test also fails for natural properties. Imagine scientists discover a deductive system for our world which by all parties concerned scores highest on the simplicity/strength bal-

2 Armstrong would disagree: according to him there are good epistemological grounds for positing $\mathrm{N}$ such as inference to the best explanation $(1983,104)$. Cohen and Callender $(2009,13)$ consider and reject whether such an appeal could be made for natural properties as well. 
ance and is also formulated in terms that refer to natural properties. Now suppose a new scientist enters the field with a new system. This system scores better than the old: it is simpler and it contains more information. However it uses a different basic set of kinds and so does not refer only to natural properties. As van Fraassen questions 'how could we designate this an evil day for science?' $(1983,53)$ The point is that scientists would consider the new system the 'best system' showing that on the criteria given by the BSA there would be no way for a scientist to know whether the best system laws really are indeed the laws of nature.

It appears therefore that there is a good motivation for Humeans to seek a version of the BSA without natural properties. Naturally, if one is happy to accept natural properties and/or reject Humeanism, then it is possible to sign up to the BSA and consider the language-dependency problem solved. The remainder of this discussion will be aimed squarely at those philosophers who, for broadly Humean reasons find the BSA attractive, but are not yet ready to admit the existence of natural properties.

\section{Adaptivist Solutions: The Package Deal Account and the Better Best System Account}

Once we have rejected natural properties, what other options remain? We need to have an agreed background of kind-distinctions from which to judge the simplicity and strength of candidate systems, but it needn't be natural properties that provide that background. One option, which Cohen and Callender call 'stipulationism' (2009, 15-20), would be to allow science to permit a once-and-for-all stipulation of acceptable terms. ${ }^{3}$ Science could then progress under the contract that all future theories and systems be couched only in terms from this 'stipulated base'. Possible suggestions for this base taxonomy could include a restriction to physical magnitudes, macroscopic properties, microscopic properties or observable properties.

Whilst acknowledging that such a stipulated base could solve the language-dependency problem, Cohen and Callender give good reasons for thinking that this is not an ideal solution (2009, 17-20). Perhaps the most important criticism Cohen and Callender give is that a lot hangs on the decision of the base and yet the decision itself seems somewhat arbitrary. When making a once-and-for-all stipulation the theoretical options available for scientific inquiry are forever restricted. Scientists cannot create new taxonomies but must constantly rework the base in light of new empirical evidence. It seems intellectually irresponsible to shut down potential pathways of inquiry from the get-go and does not seem to fit with the way science has progressed. To be sure at times scientists do object to the introduction of new kinds (for example the hostility shown towards Newton's gravitational 'action at a distance'); but that objection is always given as part of a theoretical alternative (even if that alternative system does not score very highly).

The insight here is that the selection of appropriate kinds for system comparison should be part-and-parcel of scientific inquiry and so should not be exempt from revision and evaluation. Cohen and Callender call this alternative 'flexible stipulationism'; but I

3 Cohen and Callender $(2009,16)$ cite John Earman (1986) in addition to an earlier Loewer (1996) as advocates of this kind of solution to the language-dependency problem. 
will call it 'adaptivism' as the central idea is that the language of science is allowed to adapt in light of pragmatic and explanatory pressures coming from theory selection:

With respect to the problem of arbitrariness, the proponent of stipulative [BSA] can treat her stipulated fixed background (of kinds, observable, etc.) as a pro tanto, a posteriori, and defeasible assumption that is not insulated from empirical inquiry. The thought would be that, while some or other stipulated background is needed to carry out comparisons needed to fix [BSA] laws, the background can itself be subject to rational revision on other occasions. $(2009,20)$

They make an analogy to Neurath's ship: each plank in the ship is vulnerable to replacement, but we need some planks in place from which to stand to replace the others.

Loewer advocates a similar solution in his 'package deal account' (PDA). According to Loewer we should let science make the decision about the right language. When science reaches the 'final theory' — which is judged to be the best by the scientific communitythen we can identify the language and laws together:

Here is a way of thinking of the BSA in which Lewisian natural properties play no role. Consider the world $\mathrm{W}$ of all pairs $\langle\mathrm{L}, \mathrm{T}\rangle$ of possible languages $\mathrm{L}$ and candidates for best systems of W T such that

i) $\mathrm{T}$ is formulated in $\mathrm{L}$

ii) $\mathrm{T}$ is true of $\mathrm{W}$

iii) $\mathrm{T}$ is a final theory for $\mathrm{W}$ (i.e., $\mathrm{T}$ is true and best satisfies the criteria of simplicity, informativeness, comprehensives, and whatever other conditions the scientific tradition places on a final theory for $W$.

For all such pairs $<\mathrm{L}, \mathrm{T}>$ select the one (ones) $<\mathrm{FL}, \mathrm{FT}>$ that includes the best theory [where FL refers to the language of the final theory]. $\langle\mathrm{FL}, \mathrm{FT}\rangle$ is the 'best of the best' and determines the laws of W. (Loewer 2007, 324)

Notice that there are two rounds of theory appraisal that occur in Loewer's PDA. First, theories from within a particular background of kind distinctions are compared for their theoretical merits to determine the best system for that language. This gives a class $\langle\mathrm{L}, \mathrm{T}\rangle$. Then from all such possible languages and best systems $\left.<\mathrm{L}_{1}, \mathrm{~T}_{1}>,<\mathrm{L}_{2}, \mathrm{~T}_{2}>, \ldots,<\mathrm{L}_{\mathrm{n}}, \mathrm{T}_{\mathrm{n}}\right\rangle$, select the class which is the 'best of the best' with respect to the virtues the scientific community finds desirable in a final theory. We can then define the laws of nature as the axioms in this final class $<\mathrm{FL}, \mathrm{FT}>$ as well as a language suitable for the uses that Lewis puts natural properties to.

Loewer's PDA has good theoretical economy in that in the process of selecting the final theory we get both the laws and the language together. Lewis' trivial system $(\mathrm{x})(\mathrm{Fx})$ is also dismissed in that although this does well in the first round, it fails in the second round of comparisons, since it lacks explanatory appeal from the stand-point of the scientific community.

Despite this, Loewer's PDA raises a problematic question: although it is clear how theory comparisons are made in the first round (since there is an agreement on language terms), how is this possible in the second round, when we are comparing theories in different languages? Loewer admits that in the practice of science comparisons will need to be 
relative to a linguistic base, but he thinks this can be achieved by considering the 'rational development' of a language:

A candidate for a final theory is evaluated with respect to, among other virtues, the extent to which it is formulated in SL [the language of current science] or any language SL+ that may succeed SL in the rational development of the sciences. By 'rational development' I mean developments that are considered within the scientific community to increase the simplicity, coherence, informativeness, explanatoriness, and other scientific virtues of a theory. $(2007,325)$

I find it difficult to see how this guarantees the selection of a final theory $<$ FL, FT $>$. It assumes that there will be a consensus over which $\left.\left\langle\mathrm{L}_{1}, \mathrm{~T}_{1}\right\rangle,\left\langle\mathrm{L}_{2}, \mathrm{~T}_{2}\right\rangle, \ldots,<\mathrm{L}_{\mathrm{n}}, \mathrm{T}_{\mathrm{n}}\right\rangle$ scores highest concerning the virtues-but surely this is the very point in question. Because each scientist would be advocating a different final language (FL) then each would recommend a different development of SL in the direction of their final language and their rational considerations for doing this would be relative to their background of kinds.

We have seen how simplicity and strength considerations are relative to a theory's kind distinctions, but Loewer also cites comprehensivess, informativeness and explanatoriness as additional theoretical virtues. Can these be used to help explain how scientists decide among competing systems? It is not clear how these virtues are any less dependent on language than simplicity. 'Informativeness', as we have already seen above, depends upon how one carves the world, and so would be measured differently from each linguistic stand-point. 'Comprehensivess' seems to amount to the same as 'balance' in the BSA. A good theory is one which tells us a lot about a natural phenomenon in a simple way. Hence this will also be language-dependent if simplicity and strength are. 'Explanatoriness' looks more promising; however the nature of scientific explanation is controversial and two of its most popular interpretations cannot be appealed to here. Carl Hempel's (1965) 'deductive-nomological' approach uses the concept of a law of nature in its analysis and so using this runs the risk of giving a circular analysis. Philip Kitcher's (1981) 'unificationist model' is also problematic. According to Kitcher 'a theory unifies our beliefs when it provides one (more generally, a few) pattern(s) of argument which can be used in the derivation of a large number of facts' $(1981,333)$. But clearly this idea is too close to comprehensiveness (and so also simplicity) to guarantee we have escaped the worry of language-dependency.

Cohen and Callender call their adaptivist version of the BSA the 'better best system account'. Their definition of a law differs slightly from Loewer's: whereas Loewer defines lawhood relative to the terms in the 'final theory', Cohen and Callender are more liberal. For them every best system in every language has laws and the concept of lawhood is relative to a particular background of kinds. Their view is therefore consistent with a more agnostic stance about the direction of science i.e. concerning whether it will culminate in a 'final theory' or will continue to change and develop forever.

They rule out Lewis' system $(\mathrm{x})(\mathrm{Fx})$ by saying that whilst this is a law from the perspective of the background ' $F$ ' 'it is not a best system relative to the kinds we [i.e. the scientific community] care about. If we care about mass, charge, spin, etc., then relative to these kinds, Schrodinger's equation (for example) might result, not 'all events are F" (2009, 23).

We are still left with a question concerning how the scientific community arrives at a consensus of kinds, given that different groups of scientists advocate theories with radically 
different ontologies. Cohen and Callender disagree that this leads to a radical interpretation of Kuhn's incommensurability thesis. Their response is that in reality scientists rarely work in entirely different worlds. Although they may advocate different theories and different kind-structures, there is always some overlap in their taxonomies that makes meaningful comparison possible.

Take the example of scientist A who advocates the Ptolemaic view of celestial mechanics and scientist $B$ who advocates the Copernican view.

\footnotetext{
Scientists A and B disagree, but they might agree that getting (say) the observables, suitably characterised, is important. They could then formulate the best system relative to the observables of interest, which would be a third preferred $\mathrm{X}, \mathrm{X}_{\mathrm{C}}$. At this point they can ask: is the on balance strongest system really one requiring the system of concentric spheres? $(2009,31)$
}

What Cohen and Callender say here seems convincing. Ptolemaic and Copernican scientists knew they were disagreeing about something, i.e. they understood they had different theories about how the heavenly bodies move. So there must have been some part of their vocabulary that they agreed upon. And the history of science suggests that different groups working under different theoretical assumptions do eventually decide a winner and convert the losers (at least in the natural sciences). It is not as if there still exists a community of Ptolemaic researchers engaged in trying to convince everyone else they were right all along.

The problem once again is that no clear story is given as to how this decision is supposed to be reached in practice. Cohen and Callender say they can appeal to overlapping interests; but it is not obvious how this is supposed to work. True, scientists A and B may agree in one sense that they are both describing the heavenly bodies, but each also offers a new taxonomy for describing them. If scientist-A claims their theory is the best for systematising their re-description, how will they rationally convince scientist- $B$, when $B$ is not interested in systematising A's re-description but their own? Cohen and Callender have given us no clear mechanism explaining how the overlapping virtues and taxonomies can be of any use when each scientist is aiming to re-describe and re-carve nature. It seems there would be an inevitable deadlock here in trying to convince the other.

One of the motivations for seeking an alternative to Lewis' natural properties was that his view risks making the laws epistemically inaccessible to science. Yet adaptivism is equally at risk of making the laws inaccessible. If we cannot give a clear story of how rival theories and their languages can compete and a winner be selected, then science will forever remain in deadlock concerning which is the best system. We have not yet been given a plausible account of how the virtues of a good theory (e.g. simplicity, informativeness, comprehensiveness, etc.) can be used to select rival theories when those theories make different kind distinctions in the world. Clearly scientists do make a choice, and so if adaptivism is to work, we need a more plausible story as to how this happens in practice.

\section{Interpreting Simplicity as Compression}

In the last section we saw that the main problem for current adaptivist solutions is a failure to describe any clear mechanism by which the virtues of a good theory shared by scientists, e.g. strength, simplicity, comprehensiveness, explanatoriness etc, could be made to work 
to decide among competing theories in different languages. Nevertheless, I do believe that adaptivism is the right way to tackle the language-dependency problem. The reason why current efforts struggle to make clear this mechanism is because they rely on a vague and imprecise notion of 'simplicity'. In a recent article James Woodward claims this is part of a broader problem for best system accounts. He criticises current advocates of the BSA for not drawing a closer connection between their use of simplicity and its actual role in scientific practice:

Defences of the BSA tend to treat the preference for simplicity as well as the notion of simplicity itself as a kind of primitive, and build these into the characterization of laws (laws just are generalizations that figure in systematizations best combining simplicity and strength) so that questions about the relationship between simplicity and lawfulness, and why we should value simplicity are not given non-trivial answers. $(2014,109)$

As remarked already, Lewis does not provide a definite analysis of what simplicity consists in, although he does at times seem to equate it to the number and length of the axioms. Let us call this the 'standard interpretation' of simplicity. There are many reasons to doubt whether this concept of simplicity plays the role in scientific practice that the BSA assumes it does ${ }^{4}$, not least because it leads to the problem that simplicity can be too easily had through an appropriate choice of predicates.

In this section I will argue for a different interpretation of simplicity, namely, 'simplicity as compression', and then in section 5 show how - by interpreting simplicity in this way- we can begin to get a sense of how theories can be compared and selected despite being in different languages.

The suggestion that the BSA could be improved by thinking of simplicity in terms of compression was first given by David Braddon-Mitchell $(2001)^{5}$, but the idea that an important part of scientific inquiry is the search for short, compressed descriptions of nature, goes back much further.

The concept of compression is easy to grasp. Consider the following string of digits:

$$
010101010101010101010101010101010101010101
$$

Intuitively this string of data contains a certain fixed amount of information. This string can, however, be written in a shorter way whilst retaining the same amount of information:

$$
\text { “01”(*21) }
$$

Uncontroversially, nature provides us with a source of information. Scientists are therefore in a position to decide how best to encode that information. The basic idea is that scien-

4 Woodward (2014) raises a number of important objections to the standard interpretation, finding little endorsement for it by practicing scientists and questioning whether it always needs to be traded-off against strength.

5 Braddon-Mitchell's concern was not with the language-dependency problem. For him the BSA is best thought about in terms of compression because it helps solve problems with exception-ridden laws, counterfactuals and Hume's arguments about miracles. 
tists choose to encode it in the most economic way possible, which involves looking for compressed descriptions. This is the inspiration behind Ernst Mach's $(1883,1894)$ instrumentalist view. Mach likens science to a kind of business 'consisting of the completest possible presentment of the facts with the least possible expenditure of thought' $(1883,586)$. Laws play a crucial role in this 'economic description of the world' by reducing the number of individual data points that need to be recorded or memorized:

The communication of scientific knowledge always involves description, that is, a mimetic reproduction of facts in thought, the object of which is to replace and save the trouble of new experience. Again, to save the labor $[s i c]$ of instruction and of acquisition, concise, abridged description is sought. This is really all the natural laws are. Knowing the value of the acceleration of gravity, and Galileo's laws of descent, we possess simple and compendious directions for reproducing in thought all possible motions of falling bodies. $(1894,193)$

For a given set of data, compression is usually made possible because the string itself contains a pattern or regularity. This allows us to construct an algorithm (a kind of rule) which significantly reduces the size of our encoding of the data. The discovery of such rules is one reason that has allowed computing technology to advance so rapidly in recent years since it permits ever larger amounts of information to be stored on smaller, more manageable devices.

This gives us an alternative way of thinking about the role of simplicity in the BSA. Instead of measuring simplicity as the number and length of the axioms, we can think of the best system as the one which contains the best rules for compressing empirical data. ${ }^{6}$ By 'best rules' we mean the ones that provide the shortest description of the data compared to all other alternatives. As a solution to the language-dependency problem this won't quite do, since it should be obvious that once again the length of the compression will depend on which language I choose to encode the world in the first place.

The reader at this stage might then wonder what benefit can be had from switching to compression? In section $5 \mathrm{I}$ will show how a result from algorithmic information theory known as the 'invariance theorem' can be put to use to mitigate some of these concerns. In particular, whilst it won't remove the possibility of alternatively good systems in alternative languages, it does make possible comparison and choice between scientific theories in practice, therefore avoiding the potential deadlock faced by Loewer's and Cohen and Callender's theories.

Woodward said that the problem with extant versions of the BSA is that they presuppose the value of simplicity to science and fail to give evidence for its connection to the formulation of laws. By thinking of simplicity as compression we have an answer to Woodward's first complaint: simplicity is important for descriptive economy. Presumably scientists cannot create a list of the positions and properties of every individual in the universe, even if they wanted to. This would vastly outstrip our capacity and resources. Algorithmic compression therefore makes description more manageable given that we are finite creatures working with finite resources.

What about Woodward's other concern? Even if it turns out compressibility considerations can be used to decide between competing theories, this still might not help scien-

6 Terence Tomkow (2014) also offers a theory of laws as programs for compressing data; however he sees his as an alternative to the BSA. Like Lewis though he accepts natural properties. 
tists discover the laws because scientists themselves might not actually be interested in compressing empirical data. To alleviate this worry I will now discuss two pieces of evidence that indicate compressibility is an important element in scientific practice.

The first piece of evidence is that scientists themselves seem to think about the desire for simple theories in terms of the compression of empirical data. Albert Einstein (1933) writes:

It can scarcely be denied that the supreme goal of all theory is to make the irreducible basic elements as simple and as few as possible without having to surrender the adequate representation of a single datum of experience.

Richard Feynman gives a similar sentiment: 'It is possible to condense the enormous mass of results to a large extent - that is to find laws which summarize' $(1963,1)$. Using the terminology of algorithmic information theory, the astronomer Paul Davies is even more explicit: 'The existence of regularities may be expressed by saying that the world is algorithmically compressible. Given some data set, the job of the scientist is to find a suitable compression...' (1995, 249).

The view that science is the search for algorithmic compressions of nature is most often associated with the scientist Murray Gell-Mann:

The best way to compress an account of large numbers of facts in nature is to find a correct scientific theory, which we may regard as a way of writing down in a concise form a rule that describes all the cases of a phenomena that can be observed in nature. (1987)

Interestingly, Gell-Mann does not identify scientific laws with the algorithms in the shortest description of nature. His reason is that he does not think the concepts of simplicity and complexity afforded by algorithmic information theory connect well with our ordinary uses of these terms (1994, 43-50). To see why, compare the works of Shakespeare with an output of equal length from a room of typing monkeys. The work of the monkeys will be mostly incompressible: it will not contain any repeating passages that can be summarised by a rule. Shakespeare's work will be more compressible however, as it is known that English words and sentences contain patterns. In accordance with algorithmic information theory this makes the shortest description of the typing monkeys longer than the shortest description of Shakespeare and as a result, this makes the monkeys' work more complex. Conversely, it implies that Shakespeare's work is simpler than the monkeys'-but this does not square well with our ordinary intuitions.

Gell-Mann's solution is to limit the compression only to the regularities in the data we are interested in. He calls this the 'effective complexity':

A measure that corresponds much better to what is usually meant by complexity in ordinary conversation, as well as in scientific discourse, refers not to the length of the most concise description...but to the length of a concise description of a set of the entity's regularities. Thus something almost entirely random, with practically no regularities, would have effective complexity near zero. $(1995,2)$

Since data sets contain numerous patterns-he thinks it is largely down to human interests which ones we compress and therefore which algorithms become the laws. 
This then raises a question about the use of compression being suggested here. Should we identify the laws with the algorithms in the shortest description of nature (that also produces the random incompressible parts)? Or should we identify them only (as Gell-Mann does) with the shortest description of the regularities? I don't think the decision we make here will make much difference to the overall view. This is because we can let the 'strength' desiderata already implicit in the best system account select only the algorithms for the regularities we care about. For ease of description then, I suggest sticking to the original formulation of simplicity, i.e. the shortest total description. The resulting best system will not contain all regularities-only the ones scientists care about after the strength desiderata have been applied.

The second piece of evidence is that examples of scientific laws do seem to look like they compress empirical data. Take as an example Kepler's laws of planetary motion. We could record a table of singular data for all the positions of the sun, moon and earth over a thousand years. Yet clearly we could write this more simply by just noting the position of each on day one and then work out the rest of the data using Kepler's laws. The laws here are aiding a more concise description of the data and so effectively are working as the algorithmic component in a data compression. We can extend the point further and explain the difference in the fundamentalness of laws with respect to how much compression they achieve: Newton's laws are more fundamental than Kepler's because they compress data about the motion of both celestial and terrestrial bodies.

James McAllister (2003) disagrees that scientific laws compress empirical data. According to him all known 'empirical data sets consist of random strings of digits' $(2003,634)$. Given our definition above this would make empirical data maximally complex and incompressible. This seems like an extraordinary claim as we are used to thinking of nature as being orderly and regular. He gives a number of arguments that aim to show laws do not compress empirical data. As many of these have been critically discussed at length in Twardy et al. (2005) I won't repeat them here. One of his arguments is worth looking at in more detail, however, as it potentially impacts the possibility of using compression considerations for theory comparison.

McAllister gives an argument in the form of an induction on past scientific laws: he says all previous attempts at compression have failed. This is because all scientific laws we know of cannot be used to reproduce the data with perfect accuracy. There will always be a discrepancy of some size, no matter how small, between the output of the algorithm and the empirical data. The explanation he gives is familiar. All actual physical systems are subject to a variety of impediments and interfering factors-the effects of which are often chaotic and unpredictable $(2003,637)$. This means that any rule created by the compression of some collected finite data set cannot be expected to get good results when applied to another data set for a physical system of a similar type.

There are a number of replies the advocate of compression could give here. It might be that the problem stems from the fact that we are only using finite samples of data from systems that are not 'closed-off from the effects of interfering phenomena. But we can idealize a little, as Lewis does, and imagine that when we have all the data (for the entire universe), then a suitable combination of the best algorithms will result in the correct output. Currently our efforts are limited only because of the access we have to data and our lack of knowledge of additional algorithms that would be needed in the best system to cover these additional impediments. 
Another response one could give is to recognise a distinction commonplace in computer science between lossless and lossy forms of data compression (Braddon-Mitchell, 2001). A lossless algorithm is one which produces all the original data intact after decompression: a lossy algorithm is one which acquires greater gains in compression but at the cost of accuracy in the output. For example JPEG is a lossy compressor; after the picture has been taken and recorded noticeable 'blocking artefacts' are present in the decompression reconstruction of the image. We could argue that scientific laws are lossy compressions of the empirical data-they make deliberate simplifications in order to provide greater gains in compression. This gives a new way of thinking about the simplicity/strength trade-off in the BSA: truth or accuracy in the description could also be sacrificed if the simplicity gains are good enough.

This response to McAllister does unfortunately come at a cost for it would imply the existence of more laws than many would be comfortable with. To illustrate the point, consider the following two sets of data ${ }^{7}$ :

a) 01010101

b) 10101010

Suppose we wanted to compress (a) and (b) using a lossy compression. It is clear that a number of algorithms would do the job: "01" (*4) would repeat the data correct for (a) but not for (b); "10"(*4) would repeat the data correct for (b) but for (a). Which one should we choose? It appears we would have no choice but to accept them both. Hence even before we consider the possibility of alternative encodings we are faced with equally good alternatives if allowing for lossy laws.

I don't think this is a problem specific to the BSA. If inexact laws (such as ceteris paribus laws or idealized laws) are genuine laws of nature then all accounts of lawhood would need to accommodate this. Far from being a problem for this interpretation, compression considerations give us a way to explain the lawhood of inexact scientific laws. It is true this may lead to more than we might like, but the same point could be levelled against others, such as Armstrong's theory of 'oaken laws' (Armstrong 1983, 147-150). In what follows, since my concern is with the language-dependency problem, I will limit the discussion only to 'strict laws', that is, algorithms which compress the data in a lossless way.

\section{Comparing Compression in Different Languages}

It is now time to show how, by taking compression or compressibility to be key a virtue of scientific theories, it is possible to compare the simplicity of theories against one another despite being composed of different languages.

In order to do this it will be useful to first define some important concepts from algorithmic information theory (AIT) that will provide the framework for the explanation. Let us use the symbol ' $S$ ' to refer to an arbitrary string of binary digits. The length of $S$ can then be given a numerical value; for example, if $S=$ ' 01010101 ' then the length of $S$ is

7 I am grateful to an anonymous reviewer for raising this issue with lossy compression and supplying the example. 
8 bits. Of course we are also interested in compressing $S$ and comparing the new compression to the old so as to work out how much compression has been achieved. We can measure this by using the idea of a universal computing device $U$ which is programmable-in other words, we can feed into its storage banks a program which it can apply to additional data in order to produce a desired output. In the case of compression our desired output is $S$ and the input into the universal machine will be our compressed description $T$ which is made up of two parts: $S^{\prime}$ (the compressed unstructured data) and A (an algorithm for turning $S^{\prime}$ into $S$ ). If the size of $T$ is less than $S$ for some particular $\mathrm{U}$, then we say $\mathrm{T}$ is $a$ compression of $S$.

The compression ratio is given simply by dividing the length of $T$ by $S$ : the lower the ratio, the greater the compression. We can also ask the question: what is the best possible compression of S? For any string $S$ the Kolmogorov Complexity ${ }^{8} \mathrm{~K}(\mathrm{~S})$ is defined as the shortest input on $U$ that will output $S$ and halt. Each universal machine will require an operating language for $\mathrm{T}$ which implies that $\mathrm{K}$ is relative to the language used. However a foundational result in AIT known as the 'invariance theorem' shows that this dependency is limited to a constant value ' $c$ ':

$$
\text { Invariance Theorem: }(\forall S)\left|\mathrm{K}_{\mathrm{U} 1}(S)-\mathrm{K}_{\mathrm{U} 2}(\mathrm{~S})\right| \leq \mathrm{c}
$$

What is interesting about the value ' $c$ ' is that it cannot go on indefinitely as long as the alphabets for $\mathrm{U}_{1}$ and $\mathrm{U}_{2}$ are themselves finite. This is because we can always create a new program $\mathrm{P}_{1 \text {-to-2 }}$ which translates between the languages of $\mathrm{U}_{1}$ and $\mathrm{U}_{2}$.

$$
\text { Translation Program: } \mathrm{c}=\left|\mathrm{P}_{1-\mathrm{to}-2} \mathrm{U}_{1}(\mathrm{~S}) \leftrightarrow \mathrm{U}_{2}(\mathrm{~S})\right|
$$

As ' $c$ ' is constant its effect on the value for $\mathrm{K}$ for $\mathrm{U}_{1}$ and $\mathrm{U}_{2}$ will diminish as the length of $S$ increases. Provided we are dealing with very long strings to compress, $\mathrm{c}$ becomes negligible. Many believe that this shows $\mathrm{K}$ represents something intrinsic about the complexity of an object, which does not depend on the way we choose to describe it.

At this point it might be tempting then to rely on the invariance theorem alone to solve our problem: scientists $\mathrm{A}$ and $\mathrm{B}$ are both aiming to best compress $S$, but the invariance theorem tells us the best compression of $S$ is independent of language choice. Provided the length of $S$ is large in comparison to $c$ (which is a reasonable assumption given the size of the natural world) then any language will do. If scientist-A finds out $\mathrm{T}_{1}$ is the best description of $S$ using the language of $U_{1}$ and scientist-B finds out $T_{2}$ is the best description of $S$ using the language of $U_{2}$, then it will provide little difference to each scientist whether they choose to use $T_{1}$ or $T_{2}$, since to do so requires adding only the translation program $P_{1}$. to-2 which is typically a very small addition overall.

Unfortunately, as desirable as this sounds, we cannot depend on the invariance theorem in this way to solve the language-dependency problem. One issue confronts us when applying this to Lewis' case $(\mathrm{x})(\mathrm{Fx})$. By the intuitions of all concerned $(\mathrm{x})(\mathrm{Fx})$ does not

8 AIT was developed independently by Ray Solomonoff (1964a, 1964b), Andrei Kolmogorov (1965) and Gregory Chaitin (1966). Following standard practice I will continue to refer to the algorithmic complexity of a string with the symbol ' $\mathrm{K}$ ' after Kolmogorov. 
represent a law of nature, but by the eyes of the invariance theorem it has done nothing wrong. If our universal device $\mathrm{U}$ runs in the language of ' $\mathrm{F}$ ', then $(\mathrm{x})(\mathrm{Fx})$ could well be the best compression of S. ${ }^{9}$ The reason is that ' $c$ ' can be as large as we want it to be. In a finite universe we can always artificially make our description of $S$ as simple as we want by a suitable choice of $U$ and its operating language. A way to avoid this might be to reason that the universe is infinite and so provided our language itself does not have an infinite number of characters (again a reasonable assumption) then we can safely ignore ' $c$ '. The trouble with this line of response is it requires the universe to be infinite yet the current empirical evidence on this question is inconclusive. Additionally, even if it were shown the universe is infinite in size, the resulting version of the BSA would make it conceptually necessary that laws only exist in infinite universes-yet there seems to be no a priori limitation on there being laws in a finite universe.

Another issue with relying on the invariance theorem alone is that it misrepresents the situation that gave rise to the language-dependency problem. It assumes scientists are interested in compressing one-and-the-same string of data S. But this isn't the case. As we saw above, scientists may very well want to re-describe the data using a new choice of terms. Hence from the standpoint of scientist- $A$ they will be interested in compressing $S_{A}$ whereas scientist-B will be interested in compressing $S_{B}$. Now, given that $A$ and $B$ will have their own theories $T_{A}$ and $T_{B}$, how should we compare their efforts given that they are interested in entirely different strings of data?

The first step towards answering this question is to take on board one of the assumptions of Cohen and Callender that although scientists with different theories use different taxonomies in describing the world, there is normally always agreement among scientists that they are providing alternative, competing accounts. Copernican and Ptolemaic astronomers had different perspectives concerning how to carve the world, but in broad terms, they understood that each had a different theory to explain the motions of the heavenly bodies. From this we can then say that although scientists $\mathrm{A}$ and $\mathrm{B}$ are aiming to compress different strings $\left(S_{A}\right.$ and $S_{B}$ respectively) their theories $T_{A}$ and $T_{B}$ are alternatives and therefore rival explanations of the world.

The next step is a reminder that in accordance with adaptivism language choice is partand-parcel of the attempt to provide the best system. It denies stipulationism which says there is a privileged language. Language is in a sense only a tool to reach the main aim of strong and simple theories: if it can be shown that using one language helps us achieve this goal better than another then that language should be preferred. The trouble has been with the process of showing how one language can be better than another - vis-a-vis simplicity and strength - given that these virtues are relative to each language.

Here is a proposal for how this can be achieved. It works by assuming along with AIT that we can assign a value to the length of a translation program $P$ between two languages.

Suppose scientist-A believes that $T_{A}$ is the best compression of $S_{A}$. Scientist-B likewise believes that $T_{B}$ is the best compression of the string they are interested in $S_{B}$. Now both $A$ and $\mathrm{B}$ are adaptivists and they also value compressed description of the strings they are in-

9 Strictly-speaking the algorithm here would not contain any variables, but instead would be a single command to print ' $\mathrm{F}$ ' $\mathrm{n}$ times where the value for $\mathrm{n}$ (the cardinality of the universe) is given in the unstructured (non-algorithmic) data. 
terested in. Scientist- $A$ is now in a position to compare their best theory $T_{A}$ against $T_{B}$ as a compression of $S_{A}$. Remember, $T_{B}$ was never meant to describe $S_{A}$ and so as it stands could not return $S_{A}$ on a universal machine operating in the language of $A$. But as we know there is a way to make this machine $\mathrm{U}_{\mathrm{A}}$ read $\mathrm{T}_{\mathrm{B}}$ : we just need to add an additional program $\mathrm{P}_{\mathrm{B} \text {-to-A }}$ which translates between the languages of $A$ and $B$. Scientist $A$ can then compare the sizes of two alternative inputs on $U_{A}$ that will return their data of interest $S_{A}$ :

1. $\mathrm{T}_{\mathrm{A}}$

2. $T_{B}^{A}+P_{B-\text { to-A }}$

If the size of ' $\mathrm{T}_{\mathrm{A}}$ ' is greater than the size ' $\mathrm{T}_{\mathrm{B}}+\mathrm{P}_{\mathrm{B}-\mathrm{to}-\mathrm{A}}$ ' as inputs on $\mathrm{U}_{\mathrm{A}}$ returning $\mathrm{S}_{\mathrm{A}}$, then scientist- $A$ has no choice but to accept that $T_{B}$ is a better theory.

Notice that in this explanation the standards of strength and simplicity used by scientist-A are still made from the perspective of their choice of language. The length of the best compression is measured with respect to the input in their operating system $\mathrm{U}_{\mathrm{A}}$ and the information to be compressed is the information they are interested in-namely $S_{A}$. Hence here is a way for scientist-A to be rationally compelled to accept another scientist's theory using a different language without begging the question against their own standards of strength and simplicity.

This also gives us an explanation for why in practice Lewis' system $(\mathrm{x})(\mathrm{Fx})$ is not considered viable. For all we know $(\mathrm{x})(\mathrm{Fx})$ may be the best compression of the world with respect to $U_{F}$, but we have no way of knowing this because we cannot construct a translation program between our current theories and $(\mathrm{x})(\mathrm{Fx})$. As we cannot construct such a translation program it is impossible for us to assess whether $(\mathrm{x})(\mathrm{Fx})$ is strong and simple with respect to the empirical data we care about in our current scientific vocabulary.

What if it turns out there is no difference in size between our two rival compressions? This is where the true utility of the invariance theorem comes in. It provides us with an upper bound on language choice. If the difference between $T_{A}$ and $T_{B}$ is equal to or roughly equal to the size of the translation program $\mathrm{P}_{\mathrm{A}-\mathrm{t}-\mathrm{B}}$ then we know that $\mathrm{T}_{\mathrm{A}}$ and $\mathrm{T}_{\mathrm{B}}$ are both equally good compressors and that simplicity considerations alone cannot be appealed to decide the difference. This doesn't mean that scientists-A and -B will be in a deadlock: there might be other considerations that could be brought to bear on the issue, such as how well $T_{A}$ and $T_{B}$ and their attending languages fit with other known theories that both $A$ and $\mathrm{B}$ accept.

Even if in practice such equivalence is rare, it might be objected that the mere possibility of it occurring renders the BSA problematic as an account of laws. For example, let language-A encode ' $\mathrm{o}$ ' $=\{1\}$ and ' $\mathrm{k}$ ' $=\{0\}$ and language-B encode' $\mathrm{o}$ ' $=\{0\}$ and ' $\mathrm{k}$ ' $=\{1\}$. Both languages need the same number of bits (two) to encode 'OK' yet they do so in different ways. Which encoding is correct? It could be argued that this then gives us many best systems, one for each language possible, if the systems cover the facts we are interested in but are equal in length.

I don't believe this possibility takes anything away from the explanation being offered here. The challenge this presents is the problem of ties among equally good best systems whereas we set out to solve the problem of language-dependency of strength and simplicity in theory comparison. The problem of ties is one that Lewis himself grappled with and indeed all versions of the BSA will have to answer at some point. Lewis considered two possible solutions to this problem. Firstly, we could hope that the best system is so much better than 
all alternatives that there just are no ties $(1994,479)$. Alternatively, if there are ties, then we can take the axioms common to each tied system and call these the laws $(1973,73)$.

The only additional level of complication I can foresee compressibility considerations adding to this is that Lewis' second solution may no longer be available to us. The best systems will be tied but they will also be composed of different languages hence there will be no common axioms. In this scenario the advocate of adaptivist BSA will just have to accept that there will be equally good laws in equally good languages, but the ones that matter are the ones that scientists have selected. After all, adaptivism rejects the possibility of there being a privileged language so this result is not surprising. It remains an interesting and important question why scientists choose the taxonomies that they do for their theories in the first place. Nonetheless, that is a distinct problem from the one we set out to solve which is once scientists have selected a language how is it possible to compare rival theories in alternative languages? Hopefully this paper has made some progress in showing how this is achievable.

\section{REFERENCES}

Armstrong, David. 1983. What is a Law of Nature? Cambridge: Cambridge University Press.

Braddon-Mitchell, David. 2001. Lossy Laws. Noûs 35(2): 260-277.

Chaitin, Gregory. 1966. On the Length of Programs for Computing Finite Binary sequences. Journal of the ACM 13(4): 547-569.

Cohen, Jonathan and Callender, Craig. 2009. A Better Best System Account of Lawhood. Philosophical Studies 145(1): 1-34.

Davies, Paul. 1995. Algorithmic Compressibility, Fundamental and Phenomenological Laws. In Laws of Nature: Essays on the Philosophical, Scientific and Historical Dimensions. Berlin: Walter de Gruyter \& Co.

Earman, John. 1984. Laws of Nature: The Empiricist Challenge. In D. M. Armstrong, ed. R. J. Bogdan, 191223. Dordrecht: D. Reidel Publishing Company.

Eddon, M and Meacham, C. J. G. 2015. No Work for a Theory of Universals. In The Blackwell Companion to David Lewis, eds B. Loewer \& J. Schaffer, 116-137. Oxford: Wiley-Blackwell.

Einstein, Albert. 1933, June 10. On the Method of Theoretical Physics. Oxford: The Herbert Spencer Lecture. Feynman, Richard. 1963. Lectures on Physics. Reading, MA: Addison-Wesley.

Gell-Mann, Murray., 1987, October 1. Simplicity and Complexity in the Description of Nature. Pasadena: The Caltech Associates.

-. 1994. The Quark and the Jaguar. London: Little, Brown and Company.

-. 1995. What is Complexity? Complexity, 1(1): 1-9.

Halpin, John. 2003. Scientific Law: A Perspectival Account. Erkenntnis 58: 137-168.

Hempel, Carl. 1965. Aspects of Scientific Explanation. New York: The Free Press.

Kitcher, Philip. 1981. Explanatory Unification. Philosophy of Science 48(4): 507-531.

Kolmogorov, Andrei. 1965. Three Approaches to the Definition of the Quantity of Information. Problems of Information Transmission 1(1): 1-7.

Lewis, D. 1973. Counterfactuals. Oxford: Blackwell.

—. 1983. New Work for a Theory of Universals. In: D. H. Mellor \& A. Oliver, eds. Properties. s.l.: Oxford University Press, pp. 188-227.

-. 1986. Causation. In Philosophical Papers, Volume II. Oxford: Oxford University Press.

-. 1994. Humean Supervenience Debugged. Mind 103(412): 473-490.

Li, M. and Vintanyi, P. 1993. An Introduction to Kolmogorov Complexity and its Applications. New York: Springer-Verlag. 
Loewer, Barry. 1996. Humean Supervenience. Philosophical Topics 24(1): 101-127.

-. 2007. Laws and Natural Properties. Philosophical Topics 35(1 \& 2):313-328.

Mach, Ernst. 1883. The Science of Mechanics. 1960 ed. Illinois: Open Court.

-. 1894. Popular Scientific Lectures. 1943 ed. Illinois: Open Court.

McAllister, James. 2003. Algorithmic Randomness in Empirical Data. Studies in the HIstory and Philosophy of Science 34: 633-646.

Mill, John Stuart 1895. A System of Logic. 1974 ed. London: Routledge Kegan and Paul.

Ramsey, Frank. 1990. Philosophical Papers. Cambridge: Cambridge University Press.

Roberts, John. 1999. "Laws of Nature" as an Indexical Term: A Reinterpretation of Lewis's Best-System Analysis. Philosophy of Science 66(3): 502-511.

Schrenk, Markus. 2008. A Theory for Special Science Laws. In Selected papers contributed to the sections of GAP 6, (eds) H. Bohse, K. Dreimann \& S. Walter. Paderborn: Mentis.

Solomonoff, R. 1964a. A Formal Theory of Inductive Inference: Part I. Information and Control 7(1): 1-22.

-. 1964b. A Formal Theory of Inductive Inference: Part II. Information and Control 7(2): 224-254.

Taylor, Brian. 1993. On Natural Properties in Metaphysics. Mind 102(405): 81-100.

Tomkow, T. 2013. Computation, Laws and Supervenience. [Online] Available at: www.tomkow.com

Twardy, C., Gardner, S. and Dowe, D. 2005. Empirical Data Sets are Algorithmically Compressible: Reply to McAllister? Studies in the History and Philosophy of Science 36: 391-402.

van Fraassen, Bas. 1989. Laws and Symmetry. Oxford: Clarenden Press.

Woodward, James. 2014. Simplicity in the Best Systems Account of Laws of Nature. British Journal for the Philosophy of Science 61(1): 91-123.

Billy Wheeler earned a PhD in History and Philosophy of Science at the University of Cambridge. He is currently Honorary Research Assistant in the Department for Science and Technology Studies, University College London.

Address: Department of Science and Technology Studies, University College London, Gower Street, London, WC1E 6BT, United Kingdom. Email: billy.wheeler@ucl.ac.uk 